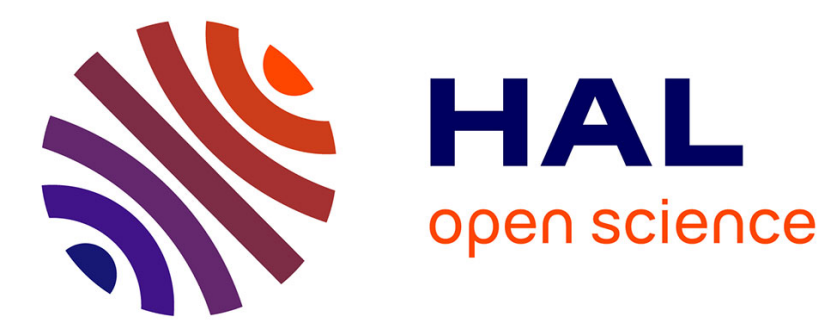

\title{
Density effects on the structure of irradiated sodium borosilicate glass: A molecular dynamics study
}

Dimitrios Kilymis, Jean-Marc Delaye, Simona Ispas

\section{To cite this version:}

Dimitrios Kilymis, Jean-Marc Delaye, Simona Ispas. Density effects on the structure of irradiated sodium borosilicate glass: A molecular dynamics study. Journal of Non-Crystalline Solids, 2016, 432, part B, pp.354. 10.1016/j.jnoncrysol.2015.10.031 . hal-01342738

\section{HAL Id: hal-01342738 \\ https://hal.science/hal-01342738}

Submitted on 11 Jul 2016

HAL is a multi-disciplinary open access archive for the deposit and dissemination of scientific research documents, whether they are published or not. The documents may come from teaching and research institutions in France or abroad, or from public or private research centers.
L'archive ouverte pluridisciplinaire HAL, est destinée au dépôt et à la diffusion de documents scientifiques de niveau recherche, publiés ou non, émanant des établissements d'enseignement et de recherche français ou étrangers, des laboratoires publics ou privés. 


\title{
Density effects on the structure of irradiated sodium borosilicate glass: a Molecular Dynamics study
}

\author{
D. A. Kilymis ${ }^{\mathrm{a}}$, J.-M. Delaye ${ }^{\mathrm{b}}$, S. Ispas ${ }^{\mathrm{a}, *}$ \\ ${ }^{a}$ Laboratoire Charles Coulomb (L2C), UMR 5221 CNRS-Univ. Montpellier, Place Eugène \\ Bataillon - CC069 F-34095 Montpellier Cedex 5 - France \\ ${ }^{b} C E A, D E N, D T C D, S E C M, F-30207$ Bagnols-sur-Cèze, France
}

\begin{abstract}
We have carried out Molecular Dynamics simulations on a sodium borosilicate glass in order to analyze how the structure of the glass during irradiation is affected by the choice of the density in the liquid state before cooling. In a pristine form generated through the usual melt-and-quench method, both short- and medium-range structures are affected by the compressive or tensile environment under which the glass model has been generated. Furthermore, Na-rich areas are much easier to compress, producing a more homogeneous glass, in terms of density, as we increase the confinement during the quench. When the glass is subjected to displacement cascades, the structural modifications saturate at a deposited energy of approximately $8 \mathrm{eV} /$ atom. Swelling appears for the glasses that were initially prepared under compression, while contraction is evident for the ones prepared under tension. We have equally prepared glass models using a fast quench method, and we have found that they present an analogous disorder as the glasses submitted to displacement cascades. Compared to the irradiated glass, we found that the magnitude of the modifications for the fast quenched glass is lower, most notably in terms of boron and sodium coordination, the percentage of non-bridging oxygens and in the ring distributions. This later result agrees with statements extracted from recent experimental works on nuclear glasses.
\end{abstract}

\footnotetext{
${ }^{*}$ Corresponding author

Email address: simona.ispas-crouzet@umontpellier.fr (S. Ispas)
}

Preprint submitted to Journal of Non-Crystalline Solids

July 11, 2016 
Keywords: borosilicate glass, molecular dynamics, irradiation effects, density

\section{Introduction}

Borosilicate glasses are of special interest to the nuclear industry, due to their use as confinement matrix for high-level waste. The R7T7 glass used in France is a complex borosilicate glass whose properties variation under irradiation has been shown to mostly depend on ballistic effects from recoil nuclei [1]. In parallel, it has been demonstrated that its behavior when subjected to irradiation can be adequately represented by more simplified glasses that contain only a few oxides [2].

The most important effect of $\alpha$-irradiation, observed in both complex and simplified glasses, is the gradual appearance of swelling and its stabilization after a certain saturation level [3, 4, 2]. Swelling is expected to reflect the changes in the glassy matrix during irradiation, most notably the depolymerization of the glass and the general increase of disorder, which cause notable changes in the mechanical properties of the glasses, especially hardness 2, 4, 5]. It can be interpreted as a result of a local melting event along the path of each displacement cascade, followed by a quench at an elevated fictive temperature [6, 7, 8, 9, 4, 10, 11]. Such similarities between the irradiated glass and the effects of the quenching temperature have been also studied experimentally by means of nuclear magnetic resonance (NMR) and Raman spectroscopies, indicating the existence of analogies between the two processes [12]. Based on these experimental results, a numerical method using a rapid quenching proposed recently has been proven to emulate the results of the displacement cascades and to be effective in terms of computational time, especially when applied to large systems where a long series of cascades would be computationally prohibitive [13, 14].

In this work we present the results of Molecular Dynamics (MD) simulations on the structural properties of a borosilicate glass (called hereafter SBN14 and equally known as CJ1) when it is cooled from different volumes in the liquid 
state. This glass composition is the best analogy to the R7T7, in terms of simplified borosilicate glasses, due to its similar $\left[\mathrm{Na}_{2} \mathrm{O}\right] /\left[\mathrm{B}_{2} \mathrm{O}_{3}\right]$ molar ratio. After the cooling to room temprature, we have launched a series of displacement cascades for each glass in order to analyze the role of density during the irradiation process. Alongside, we have prepared a glass by means of rapid quenching method and we have compared the structures generated using the two methods.

The paper is organized as follows. In Section 2 we describe the computational methodology used, while Section 3 contains the results of the simulations. We first describe the dependence of the pristine glass structure on the choice of the initial liquid volume, prior to quenching. Then, we present the effects of displacement cascades on the glass prepared with different initial liquid volumes, followed by the description of the glass structure obtained using fast quenching. Section 4 contains the discussion and Section 5 the conclusions.

\section{Computational details}

The SBN14 glass has a composition of $67.73 \% \mathrm{SiO}_{2}, 18.04 \% \mathrm{~B}_{2} \mathrm{O}_{3}$ and $14.23 \% \mathrm{Na}_{2} \mathrm{O}$ and an experimental density of $2.45 \mathrm{~g} / \mathrm{cm}^{3}[15]$. In order to fabricate the glass, we started with a random distribution of 10.000 atoms heated at $5000 \mathrm{~K}$ and reproducing the stoichiometry, namely 2015 silicon, 6064 oxygen, 1074 boron and 847 sodium atoms. The liquid was equilibrated at $5000 \mathrm{~K}$ for 100 ps using the constant volume-constant temperature ensemble (NVT), and was then cooled to room temperature using the same ensemble and a quench rate of $5 \times 10^{12} \mathrm{~K} / \mathrm{s}$. At room temperature, we switched to the constant pressureconstant temperature ensemble (NPT) with the pressure set to zero and the box size was allowed to relax for 20 ps. It should be stressed that the glasses are expected to contract during this stage and thus the density after cooling will be higher than the one of the liquid. The NPT run was followed by a production run of $5 \mathrm{ps}$ in the constant volume-constant energy ensemble (NVE). We prepared six glasses with different initial volumes in the liquid state in order to investigate how the density of the liquid affects the structure after cooling. We 
chose values of the initial liquid volume varying between $-30 \%$ to $20 \%$, when compared to the experimental volume of the glass, and with $10 \%$ increments. Further on they will be referred to by the value $V_{l}$, defined as the liquid volume over the experimental volume of the glass. These six glass structures correspond to the form called pristine glass.

The six glass structures were then submitted to a series of displacement cascades. For each cascade, one Si atom was given a high mass and launched along the main diagonal of the computational cell, with a $0.8 \mathrm{keV}$ initial kinetic energy. Throughout the phase of the cascades, the NVE ensemble was used, while a $1.5 \AA$ layer along the border of the cell was thermostatted at $293 \mathrm{~K}$. In total, 190 cascades were launched in order to reach a deposited energy of approximately $15 \mathrm{eV} /$ atom. After the cascades, the simulation cell was equilibrated for $50 \mathrm{ps}$ using the NPT ensemble in order to relax any accumulated pressure, and it was followed by a 10 ps production run using NVE.

To prepare the fast quenched glass form, we firstly selected the pristine glass exhibiting the lowest potential energy. We then launched a series of calculations where this structure was heated to temperatures varying between $2000 \mathrm{~K}$ and $3000 \mathrm{~K}$, and then relaxed for 50 ps using the NPT ensemble with a zero target pressure. The glasses were then cooled down to $300 \mathrm{~K}$ with a quench rate of $10^{14} \mathrm{~K} / \mathrm{s}$ and the NPT ensemble at zero pressure. At room temperature, further runs were carried out for 20 ps and 5 ps using the NPT and NVE ensemble, respectively. Concerning the employed quench rates for both the pristine and fast quenched glass, it should be noted that, although they are much faster than the ones encountered in experiments, they have been successfully used in the past to model the structure of sodium borosilicates [15, 13, 14, 16].

All calculations have been performed using a modified version of the DL_POLY 3.0 Molecular Dynamics code [17] with the interatomic pair potential for sodium borosilicates proposed by Kieu et al. [15]. The potential has a BuckinghamCoulomb form: 


$$
\Phi\left(r_{\alpha \beta}\right)=A_{\alpha \beta} \exp \left(-\frac{r_{\alpha \beta}}{\rho_{\alpha \beta}}\right)-\frac{C_{\alpha \beta}}{r_{\alpha \beta}^{6}}+\frac{q_{\alpha} q_{\beta} e^{2}}{r_{\alpha \beta}}
$$

where $\alpha, \beta \in\{\mathrm{Si}, \mathrm{B}, \mathrm{O}, \mathrm{Na}\}, r_{\alpha \beta}$ is the distance between atoms of type $\alpha$ and $\beta$. The effective charges of type $\alpha$ and $\beta$ atoms are given by $q_{\alpha}$ and $q_{\beta}$, while $A_{\alpha \beta}$, $\rho_{\alpha \beta}$ and $C_{\alpha \beta}$ are the rest of the potential parameters. In this potential, the effective charges, as well as the $A_{B-O}$ parameter, are adjustable. The values used in this work are given in Table 1

\begin{tabular}{lc}
\hline Parameter & Value \\
\hline q'Si & 1.868456 \\
q'B & 1.596549 \\
q'O & -0.966544 \\
q'Na & 0.450956 \\
$\mathrm{~A}_{B-O}(\mathrm{eV})$ & 229700.881498 \\
\hline
\end{tabular}

Table 1: Interatomic potential parameters used in this work.

For short-range interactions between the projectile and the glass atoms the Ziegler-Biersack-Littmark (ZBL) potential [18] was employed alongside appropriate high-order polynomials linking it to the corresponding long-range potentials, ensuring continuity of the energy, forces and force derivatives. The integration step used was equal to $1 \mathrm{fs}$, except in the phase of the displacement cascades where it was variable, down to 0.02 fs for the initial phase of each cascade. Throughout the simulations, Berendsen thermostats and barostats were used [19].

Average coordination numbers for each atom type $\mathrm{X}(\mathrm{X}=\mathrm{Si}, \mathrm{B}, \mathrm{O}, \mathrm{Na})$ were calculated by taking as cutoff distance the first minimum of the corresponding XO Radial Distribution Function (RDF). The local density values were calculated in a spherical region with a radius of $8 \AA$ around each atom. 


\begin{tabular}{lcccccc}
\hline$V_{l}$ & 0.7 & 0.8 & 0.9 & 1.0 & 1.1 & 1.2 \\
\hline Density $\left(\mathrm{g} / \mathrm{cm}^{3}\right)$ & 3.03 & 2.90 & 2.72 & 2.56 & 2.42 & 2.25 \\
$\mathrm{C}_{B}$ & 3.83 & 3.86 & 3.82 & 3.75 & 3.69 & 3.65 \\
$\mathrm{C}_{N a}$ & 9.98 & 9.97 & 9.50 & 8.73 & 7.74 & 6.94 \\
$\% \mathrm{NBO}$ & 4.16 & 2.31 & 1.75 & 2.28 & 3.17 & 3.83 \\
$\%{ }^{[3]} \mathrm{O}$ & 4.82 & 3.61 & 2.32 & 1.66 & 1.47 & 1.41 \\
\hline
\end{tabular}

Table 2: Density, B and Na average coordinations as well as the percentages of NBO and ${ }^{[3]} \mathrm{O}$ of the glass structures generated using varying $V_{l}$ initial liquid volume.

\section{Results}

\subsection{Dependence of the glass structure on the initial liquid volume}

Some structural characteristics of the six glasses prepared with different $V_{l}$ initial liquid volume are given in Table 2. As expected, effects of confinement for the glasses prepared with a low initial volume are evidenced in the B and $\mathrm{Na}$ average coordination numbers and the percentage of ${ }^{[3]} \mathrm{O}$, which increase as we decrease the initial volume. On the contrary, Si remains perfectly tetracoordinated in all cases, a fact that can be attributed to the high rigidity of the $\mathrm{SiO}_{4}$ units in the glass. Also, the percentage of non-bridging oxygen (NBO) does not follow a linear trend but exhibits a minimum value present in the glass with $V_{l}=0.9$.

Concerning the final density, we can see that it best reproduces the experimental value for $V_{l}=1.1$, due to the swelling for small $V_{l}$ and contraction for large $V_{l}$ at the time of the quench. Furthermore if we inspect the evolution of the potential energy for the different glasses, we observe that the minimum energy structure lies towards lower values, at $V_{l}=0.9$ (see Figure 1). A similar result has been recently reported by Jolley et al. for a borosilicate glass with a similar composition (called SBN12 glass in some works), where it has been pointed out that the current potential does not predict the glass with the experimental density to lie at the minimum of the potential energy curve [20].

In Figure 2 we present the radial distribution functions (RDF) for the glasses 